\title{
ESTUDOS PSICOMÉTRICOS DA ESCALA DE MOTIVAÇÃO PARA A APRENDIZAGEM DE UNIVERSITÁRIOS
}

\author{
Acácia Aparecida Angeli dos Santos ${ }^{I,}{ }^{\star}$; Adriana Rosecler AlcaráII \\ Rita da Penha Campos Zenorini ${ }^{I}$ \\ ${ }^{I}$ Universidade São Francisco, Itatiba, SP, Brasil; \\ ${ }^{I I}$ Universidade Estadual de Londrina, Londrina, PR, Brasil
}

\begin{abstract}
Resumo
Este estudo teve como objetivo buscar evidências de validade, por meio da análise da estrutura interna dos itens, para a Escala de Motivação para a Aprendizagem de Universitários e investigar a consistência interna e diferenças em relação às variáveis sexo, faixa etária e área do conhecimento. Participaram 429 alunos, de uma universidade particular do interior paulista, com idade entre 18 a 44 anos. O instrumento utilizado foi a Escala de Motivação para a Aprendizagem de Universitários. Os resultados apontaram o agrupamento dos itens da escala em três fatores, correspondentes à meta performance-evitação, performanceaproximação e aprender, que explicaram $39,41 \%$ da variância. A consistência interna dos três fatores variou de 0,72 a 0,83. Na comparação entre metas $e$ sexo verificou-se uma diferença significativa na meta performance-aproximação. Quanto à faixa etária houve diferença na meta aprender. Os resultados mostraram indices aceitáveis de validade e precisão, no entanto, novos estudos são necessários para ampliar o conhecimento sobre as características psicométricas da escala.
\end{abstract}

Palavras-chave: motivação acadêmica; metas de realização; meta aprender; meta performance

\section{Psychometric STUdy OF THE UNIVERSITY'S LEARNING MOTIVATION SCALE}

\begin{abstract}
This study objective was to search evidence of validity by items internal structure analysis to the University's Learning Motivation Scale, as well as to investigate the internal consistency and differences between gender, age and area of expertise. The participants consisted of 429 students from a private university in São Paulo, with ages ranging from 18 to 44 . The instrument used was the University's Learning Motivation Scale. The results showed that the scale items got into three factors, which are performance-avoidance goal, performance-approach goal and learning goal. These factors, explained $39.41 \%$ of the scale variance. The three factors'

\footnotetext{
^ Bolsista de Produtividade do CNPq. Endereço para correspondência: Universidade São Francisco - Itatiba, Faculdade de Psicologia, Programa de Pós Graduação Stricto Sensu Em Psicologia. Rua Alexandre Rodrigues Barbosa, 45 - Centro - Itatiba, SP - Brasil. CEP: 13251-900.

E-mail: acacia.angeli@gmail.com, adrianaalcara@sercomtel.com.br, rzenorini@gmail.com.
} 
internal consistency ranged from 0.72 to 0.83 . Comparing goals in relation to gender the findings showed a significant difference in performance-approach goal. Related to age there was differences found in learning goal. These results showed that the levels of validity and reliability are acceptable, however, further studies are needed to increase knowledge about the scale psychometric characteristics.

Keywords: academic motivation; achievement goals; learning goal; performance goal

\section{INTRODUÇÃo}

A preocupação com a motivação no ambiente educacional tem sido evidenciada cada vez mais, considerando o crescente número de pesquisas sobre o tema nessas últimas décadas. Boruchovitch e Bzuneck (2010) afirmam que as pesquisas sobre a motivação escolar têm aumentado mundialmente, sendo que o construto vem sendo estudado sob diferentes abordagens teóricas, mostrando a complexidade do assunto.

A motivação tem implicação direta com o envolvimento do aluno no processo de aprendizagem. Segundo Guimarães, Bzuneck e Sanches (2002), a compreensão dos fatores relacionados ao envolvimento dos alunos com a aprendizagem é uma tarefa bastante complexa, devido à diversidade dos elementos presentes na situação. Dessa forma, os autores visualizam a motivação como uma variável interveniente, que inclui tanto elementos internos como situacionais e possibilita o entendimento do envolvimento individual.

A importância da motivação para a aprendizagem também é ressaltada por Clayton, Blumberg e Auld (2010), quando afirmam que a motivação tem sido consistentemente associada a uma aprendizagem bem sucedida. De acordo com os autores, a compreensão da motivação dos alunos é o caminho para a promoção da aprendizagem efetiva. Nessa mesma linha, Steinmayr e Spinath (2009) evidenciam que conhecer os motivos e as metas que levam os alunos a envolver-se ou não com a aprendizagem é importante, tanto do ponto de vista motivacional, quanto ao fato de ser uma questão- chave para ajudar a compreender os processos de aprendizagem e as variáveis que os determinam.

Conforme já ressaltado, os estudos da motivação têm sido desenvolvidos na perspectiva de diversas teorias, dentre elas, a Teoria da Autodeterminação (RYAN; DECI, 2000) que focaliza a motivação intrínseca e extrínseca; a Teoria de Atribuição de Causalidade (WEINER, 1985) que aborda as crenças pessoais e sua influência sobre as causas de determinados comportamentos; a Expectativa-Valor (VROOM, 1964) que estuda as crenças, os valores e as expectativas da pessoa em relação a uma atividade; a Teoria de Metas de Realização (AMES, 1992) que diz respeito à meta ou objetivo que a pessoa utiliza para realizar uma determinada atividade, adotada no presente trabalho.

As metas de realização foram definidas por Ames (1992) como um conjunto de pensamentos, crenças, propósitos e emoções que traduzem as expectativas dos alunos em relação a determinadas tarefas que devem ser executadas. Ou seja: 
as metas são representadas por modos diferentes de enfrentar as tarefas acadêmicas. Dessa forma, a meta que a pessoa adota representa o motivo pelo qual ela realizará determinada tarefa. Clayton, Blumberg e Auld (2010) complementam essa ideia, afirmando que as metas de realização se referem às razões ou propósitos da pessoa para se engajar nas atividades acadêmicas.

As primeiras pesquisas desenvolvidas na perspectiva da Teoria de Metas de Realização descrevem apenas dois tipos de metas, aprender e performance. Quando orientado para a meta aprender o aluno busca o crescimento intelectual, valoriza o esforço pessoal, enfrenta os desafios, é persistente em relação às atividades acadêmicas e tende a utilizar estratégias de aprendizagem mais efetivas (AMES, 1992; CLAYTON; BLUMBERG; AULD, 2010; MIDDLETON; MIDGLEY, 1997; ZENORINI; SANTOS, 2010b). Os alunos com esse tipo de meta compreendem que o sucesso nas realizações acadêmicas consiste em aprimorar os conhecimentos e habilidades, progredir e dominar com criatividade os conteúdos. $\mathrm{O}$ esforço empenhado nas atividades promove orgulho e realização, enquanto que as situações de fracassos e erros são estímulos para a busca de novas estratégias para atingir os objetivos (BZUNECK, 2004). Em contrapartida, os alunos orientados para a meta performance estão preocupados em demonstrar a sua capacidade perante os outros; são menos engajados e evitam desafios. Quando em situação de fracasso, atribuem esse resultado à falta de capacidade e apresentam emoções negativas, tais como vergonha e raiva (ARCHER, 1994; BZUNECK, 2004; ZENORINI; SANTOS, 2010b).

Bzuneck (1999) afirma que embora os tipos de metas tenham características contrastantes, o aluno pode, de forma simultânea e em diferentes graus, apresentar uma orientação para as metas aprender e performance. Esse é um indicativo de que em determinadas situações a meta performance pode apresentar aspectos positivos. Nessa perspectiva, Pintrich e Schunk (1996) afirmam que na ausência da orientação para meta aprender, a preocupação com uma boa performance conduz à aplicação de esforço e ao uso de estratégias cognitivas de aprendizagem.

A possibilidade de vislumbrar aspectos positivos da meta performance foi demonstrada nos estudos de Elliot e Church (1997), Elliot e Harackiewcz (1996), Elliot, McGregor e Gable (1999), entre outros, principalmente quando acompanhada da meta aprender. Os resultados desses estudos mostraram dois componentes independentes na meta performance : aproximação e evitação. Na meta performance-aproximação o aluno busca parecer inteligente e quer estar entre os melhores da classe, enquanto que na meta performance-evitação o aluno evita qualquer situação que possa mostrar a sua incapacidade. Dessa forma, as pesquisas mais recentes incluem na meta performance, os componentes aproximação e evitação.

Igualmente, os resultados das pesquisas de Valle et al. (2006) e de Wolters, Yu e Pintrich (1996) também mostraram a diferenciação entre as tendências de aproximação e evitação na perspectiva das metas performance. Vale ressaltar, 
contudo, que a distinção entre esses dois tipos de metas tem instigado mais trabalhos de pesquisas e levado os estudiosos a reconsiderar os efeitos prejudiciais da meta performance-aproximação (VALLE et al., 2009).

No Brasil, existem algumas pesquisas que focalizaram a análise da motivação na perspectiva da Teoria de Metas de Realização. Dentre elas podem-se destacar as de Cardoso (2002), Stefano (2002), Zenorini e Santos (2004), entre outras. Essas pesquisas foram desenvolvidas no contexto universitário e entre os seus objetivos estavam o de relacionar as metas com o uso de estratégias de aprendizagem. De forma geral, os resultados indicaram que os alunos orientados para a meta aprender utilizam mais as estratégias de aprendizagem cognitivas e metacognitivas do que os que adotam a meta performance.

Quanto às pesquisas que utilizaram a Teoria de Metas de Realização com as três dimensões - meta aprender, meta performance-aproximação e meta performance-evitação - pode-se mencionar Zenorini, Santos e Bueno (2003), que realizaram um estudo com 189 universitários, com o objetivo de estabelecer evidências de validade para um instrumento que avalia a motivação na abordagem da Teoria de Metas de Realização, denominado de Escala de Sensibilidade às Diferentes Metas de Realização, originalmente desenvolvido por Midgley et al. (1998). Na análise fatorial, as metas se agruparam diferentemente do previsto, separando os itens em apenas dois fatores, meta aprender e meta performance. De acordo com os autores, uma possível explicação para esses dados pode estar relacionada à falta de percepção dos participantes da pesquisa em relação à diferença dos componentes aproximação e evitação da meta performance. No entanto, os resultados encontrados foram coerentes com os de outras pesquisas, realizadas no exterior. A análise dos dados apontou um índice de consistência interna de $\alpha=0,72$ para a meta aprender e $\alpha=0,84$ para a meta performance. Outro resultado foi a correlação positiva de magnitude forte entre a meta aprender e o uso de estratégias de aprendizagem mais complexas, como as de regulação, monitoramento e planejamento. Já com o desempenho acadêmico dos alunos, a meta aprender não apresentou correlação significativa. Além disso, os dados indicaram que as participantes do sexo feminino mostraram-se mais orientadas à meta aprender, bem como utilizavam com maior frequência as estratégias de aprendizagem e apresentaram melhor rendimento acadêmico.

A comparação dos tipos de metas entre os sexos também foi explorada nos estudos de Middleton e Midgley (1997), Midgley, Kaplan e Middleton (2001), Zenorini e Santos (2004) e Zenorini, Santos e Magalhães (2011), que constataram que os participantes do sexo masculino estavam mais orientados à meta performance. Ainda, no que se refere às diferenças nas orientações motivacionais entre homens e mulheres, podem-se mencionar os estudos de Boruchovitch (2008), Guimarães, Bzuneck e Sanches (2002) e Sobral (2003), que, embora tenham sido desenvolvidos em outra perspectiva teórica, mostraram que estudantes universitários do sexo feminino, em geral, apresentavam médias mais altas em relação à motivação intrínseca e a aplicação de esforço. Vale mencionar que o estudante orientado para a meta aprender assemelha-se ao estudante com motivação intrínseca. 
Assim como a diferença entre os sexos, a faixa etária também tem sido uma variável explorada nos estudos da motivação. Guimarães, Bzuneck e Sanches (2002) ao analisar o esforço alegado e a motivação intrínseca, verificaram que os alunos mais velhos (31 anos ou mais) apresentaram médias superiores em relação aos mais novos. Da mesma forma, Boruchovitch (2008) também constatou que a motivação aumentou com o avançar da idade. Em contrapartida, os estudos de Lepper, Corpus e Yengar (2005), Otis, Grouzet e Pelletier (2005) e Stipek (1998) mostraram uma diminuição da motivação conforme os alunos avançam na idade e na escolaridade.

Uma pesquisa que investigou a estrutura fatorial, a precisão e as correlações entre os fatores da Escala de Sensibilidade às Diferentes Metas de Realização foi a de Bueno et al. (2007). Participaram do estudo 156 alunos de Psicologia, ambos os sexos, com 22,3 anos em média $(D P=4,5)$, de duas universidades paulistas. A análise fatorial apontou quatro fatores com consistência interna, variando de 0,57 a 0,86 , sendo que o primeiro fator correlacionou-se à meta performance-aproximanção, o segundo à meta aprender, o terceiro e o quarto a aspectos distintos da meta performance-evitação. Os resultados reforçaram a ideia de que as metas de realização podem ser estruturadas em meta aprender, meta performance-aproximação e performance-evitação, porém os autores sugeriram a necessidade de novos estudos, para ampliar a compreensão em relação aos itens agrupados no Fator três que dizem respeito a "considerar importante evitar uma consequência negativa" e os do Fator quatro que se referiam a "evitar uma ação que pudesse trazer uma consequência negativa".

Zenorini e Santos (2010a) destacam que os estudos desenvolvidos com base na Teoria de Metas de Realização têm buscado compreender qual a percepção dos alunos acerca de si próprios, por que se envolvem em determinadas tarefas e por que buscam atingir determinados objetivos acadêmicos. Em busca de mais dados para explicar essas questões, os estudos que visam à construção de instrumentos para avaliar a motivação têm sido numerosos no exterior (ANDERMAN; MAEHR, 1994; MAEHR; MIDGLEY, 1991; WEINER, 1990, entre outros). No Brasil há grande carência de pesquisas nessa área.

Nessa perspectiva, Zenorini e Santos (2010a) realizaram um estudo com o objetivo de construir uma Escala de Motivação para a Aprendizagem (EMAPRE). A pesquisa foi desenvolvida em duas etapas. A primeira consistiu na construção da escala com 67 itens, elaborados com base na Teoria de Metas de Realização e na Escala de Sensibilidade às Diferentes Metas de Realização. Esses itens foram submetidos a 11 juízes com conhecimento sobre as teorias de motivação e de psicometria. Após a análise desses juízes, as autoras optaram por excluir alguns itens, passando a escala a conter 50 itens. Essa nova versão da escala foi submetida à análise semântica de 19 alunos, sendo que nenhuma dificuldade foi apontada pelos participantes. A segunda etapa da pesquisa teve como objetivo identificar as propriedades psicométricas da escala, por meio da análise fatorial e análise da consistência interna dos itens. Para tanto, as autoras realizaram um estudo com 739 alunos do ensino médio de nove escolas, sendo 559 de escolas públicas e 180 
de escolas particulares. O instrumento utilizado nessa pesquisa foi a escala com 50 itens. No entanto, os resultados dos dados submetidos à análise dos componentes principais e à rotação Varimax indicaram a exclusão de alguns dos itens, passando a versão final da escala a conter 28 itens, com três fatores, que explicaram $36,11 \%$ da variância. As cargas fatoriais das três subescalas, categorizadas em meta aprender, meta performance-aproximação e meta performance-evitação, tiveram uma variação de 0,45 a 0,71 , indicando uma grande prevalência de itens com carga fatorial satisfatória. A consistência interna dessas três subescalas, medida pelo alfa de Cronbach, apresentou índices de 0,80, 0,76 e 0,74, para meta aprender, meta performance-aproximação e meta performance-evitação.

A partir desse estudo, as autoras desenvolveram uma nova versão da Escala de Motivação para a Aprendizagem (EMAPRE-U), com 28 itens, para ser aplicada em amostras universitárias. Para essa nova escala, foram realizadas algumas adaptações quanto ao tipo de linguagem utilizada em itens, como a substituição dos termos 'tarefas escolares' para 'tarefas acadêmicas'. No entanto, essa versão da escala, denominada de Escala de Motivação para a Aprendizagem de Universitários (EMAPRE-U) ainda não possui evidências de validade relativas à estrutura interna dos itens, necessitando assim de estudos com esse propósito.

Aliado a isso, Zenorini e Santos (2010b), a partir de um levantamento de pesquisas brasileiras que focalizaram a motivação na perspectiva da Teoria de Metas de Realização, constataram que muitos desses estudos avaliam a motivação por meio de medidas que ainda não têm evidências de validade estabelecidas no Brasil. Isso evidencia a importância de se desenvolver pesquisas que visem à validação de instrumentos para a avaliação da motivação no contexto educacional.

Dessa forma, o objetivo deste trabalho é buscar evidências de validade, por meio da análise da estrutura interna dos itens, para a EMAPRE-U. Além disso, pretende-se estudar a consistência interna pelo método de Cronbach e investigar se há diferenças entre as médias dos escores em relação às variáveis sexo, faixa etária e área do conhecimento dos cursos (exatas, humanas e biológicas).

\section{Método}

\section{Participantes}

A amostra deste estudo foi constituída por 429 alunos regularmente matriculados em diversos cursos, das áreas de Exatas, Humanas e Biológicas, de uma universidade particular do interior paulista. Do total de alunos, 172 $(40,1 \%)$ eram do sexo masculino e $257(59,9 \%)$ do sexo feminino. A idade variou entre 18 anos a 44 anos $(\mathrm{M}=22,7 ; \mathrm{DP}=4,85)$. Na distribuição por áreas do conhecimento, houve predominância para a área de Ciências Exatas, com frequência de 235 (54,8\%), seguida das Ciências Humanas, com 116 (27\%) e Ciências Biológicas com 78 (18,2\%). 


\section{Instrumento}

A Escala de Motivação para a Aprendizagem em Universitários (EMAPRE-U), de Zenorini e Santos (2008) contém 28 itens e foi construída tendo como base a Teoria de Metas de Realização. Esses itens foram estruturados numa escala Likert com três opções de resposta, a saber, concordo (3 pontos), não sei ( 2 pontos) e discordo (1 ponto), sendo que a pontuação variou de 28 a 84 pontos. Os 28 itens estão divididos da seguinte forma: 12 para meta aprender, sete para a performance-aproximação e nove para a performance-evitação. A seguir, alguns exemplos de itens que compõem o instrumento: meta aprender (Faço minhas tarefas porque estou interessado nelas), meta performance-aproximação (É importante para mim fazer as tarefas melhor que os meus colegas) e meta performance-evitação (Não participo dos debates em sala de aula, porque não quero que os colegas riam de mim).

\section{Procedimentos}

A partir da aprovação da pesquisa pelo Comitê de Ética, foi solicitado aos coordenadores dos cursos, bem como a professores que ministravam aulas nos dias da coleta, a autorização para a realização da pesquisa. Dessa forma, os dados foram coletados em situação de sala de aula nos horários de funcionamento dos cursos, após a explicação dos objetivos da pesquisa e preenchimento do termo de consentimento livre e esclarecido por todos os alunos que se dispuseram a participar do estudo. O tempo médio de aplicação foi de 25 minutos.

\section{Resultados}

Inicialmente foi realizada a medida de adequação da amostra de Kaiser-Meyer-Olkin $(K M O=0,84)$ e o Teste de Esfericidade de Bartlett $\left(\chi^{2}=3273,966\right.$; $g l=378 ; p<0,001)$. Os resultados desses procedimentos indicaram que a amostra é adequada para a realização da análise fatorial, bem como apresenta índices apropriados para a correlação entre os itens.

Na sequência, os dados foram submetidos à análise fatorial exploratória, com extração dos fatores por análise dos componentes principais e rotação Varimax. Foram obtidos três fatores com autovalor acima de 2, que explicaram $39,41 \%$ da variabilidade total dos dados. Estabeleceu-se como critério o valor de corte de 0,30 . Na Tabela 1 pode-se visualizar a distribuição dos itens por fator. 
Tabela 1 - Matriz de itens com as cargas fatoriais

\begin{tabular}{|c|c|c|c|}
\hline Itens & Fator 1 & Fator 2 & Fator 3 \\
\hline Item 27 & 0.78 & & \\
\hline Item 16 & 0,75 & & \\
\hline Item 9 & 0,75 & & \\
\hline Item 26 & 0,71 & & \\
\hline Item 22 & 0,71 & & \\
\hline Item 6 & 0,60 & & \\
\hline Item 18 & 0,54 & & \\
\hline Item 8 & & 0,80 & \\
\hline Item 4 & & 0,74 & \\
\hline Item 3 & & 0,72 & \\
\hline Item 11 & & 0,67 & \\
\hline Item 15 & & 0,60 & \\
\hline Item 13 & 0,32 & 0,51 & \\
\hline Item 17 & 0,40 & 0,49 & \\
\hline Item 24 & 0,42 & 0,46 & \\
\hline Item 20 & & 0,41 & \\
\hline Item 10 & & & 0,66 \\
\hline Item 12 & & & 0,65 \\
\hline Item 5 & & & 0,63 \\
\hline Item 14 & & & 0,54 \\
\hline Item 25 & & & 0,54 \\
\hline Item 21 & & & 0,53 \\
\hline Item 23 & & & 0,51 \\
\hline Item 19 & & & 0,47 \\
\hline Item 7 & & & 0,44 \\
\hline Item 1 & & & 0,37 \\
\hline Item 28 & & & 0,34 \\
\hline Item 2 & & & 0,33 \\
\hline Autovalores & 5,49 & 3,26 & 2,29 \\
\hline Coeficiente de Alfa de Cronbach & 0,83 & 0,82 & 0,72 \\
\hline
\end{tabular}

O primeiro fator, meta performance-evitação, com autovalor igual a 5,49, explicou $19,60 \%$ da variância total por meio de dez itens, sendo que as cargas fatoriais variaram de 0,32 a 0,78 . O segundo fator, meta performance-aproximação, apresentou autovalor igual a 3,26 e explicou $11,63 \%$ da variância total por meio de nove itens, sendo que as cargas fatoriais variaram de 0,41 a 0,80 . Já o terceiro fator, meta aprender, com autovalor igual a 2,29, explicou $8,18 \%$ da variância total por meio de doze itens, sendo que as cargas fatoriais variaram de 0,33 a 0,66. A consistência interna dos três fatores foi medida pelo alfa de Cronbach. Para o Fator 1, o coeficiente obtido foi de 0,83; para o Fator 2 foi 0,82, para o Fator 3, 0,72 e para a escala total 0,82 . 
Foi também realizada a análise correlacional entre os três fatores obtidos na análise fatorial, para identificar evidência de validade de construto das subescalas. Os resultados estão na Tabela 2.

Tabela 2 - Coeficientes de Correlação de Pearson entre as três subescalas organizadas de acordo com os resultados da análise fatorial

\begin{tabular}{lccc}
\hline Subescalas & & $\begin{array}{r}\text { Meta performance- } \\
\text { evitacão }\end{array}$ & $\begin{array}{c}\text { Meta performance- } \\
\text { aproximacão }\end{array}$ \\
\hline Meta performance-aproximacão & $r$ & 0.41 & \\
& $p$ & 0.001 & \\
Meta aprender & $r$ & -0.02 & 0.13 \\
& $p$ & 0.638 & 0.006 \\
\hline
\end{tabular}

Os resultados apresentados na Tabela 2 indicam que houve uma correlação positiva moderada entre o Fator 1 - meta performance-evitação e o Fator 2 - meta performance-aproximação. Já a meta aprender apresentou uma correlação de baixa magnitude com a meta performance-aproximação e correlação nula com a meta performance-evitação.

Em seguida, foi realizada uma análise para verificar se havia diferença entre as médias obtidas, considerando as variáveis sexo, faixa etária e a área do conhecimento. No que se refere à variável sexo, esse procedimento foi realizado por meio do teste de comparação de médias $t$ de Student. Os resultados obtidos encontram-se na Tabela 3.

Tabela 3 - Resultados da comparação das pontuações médias em relação à variável sexo

\begin{tabular}{lcccccc}
\hline Fator & Sexo & $\mathrm{N}$ & Média & Desvio Padrão & $T$ & $p$ \\
\hline Meta performance-evitação & $\mathrm{M}$ & 172 & 18,83 & 3,02 & 0,20 & 0,84 \\
& $\mathrm{~F}$ & 248 & 18,76 & 3,44 & & \\
Meta performance-aproximação & $\mathrm{M}$ & 170 & 20,88 & 4,52 & $-2,28$ & 0,02 \\
& $\mathrm{~F}$ & 247 & 21,92 & 4,62 & & \\
Meta aprender & $\mathrm{M}$ & 169 & 15,90 & 3,48 & 1,45 & 0,15 \\
& $\mathrm{~F}$ & 251 & 15,39 & 3,55 & & \\
\hline
\end{tabular}

Conforme se pode verificar na Tabela 3, apenas na meta performance-aproximação houve diferença estatisticamente significativa $(p=0,02)$. Observou-se, então que as mulheres obtiveram pontuações médias significativamente superiores às dos homens nesse fator.

Para a verificação de possíveis diferenças entre as variáveis faixa etária e área do conhecimento, o procedimento utilizado foi a Análise de Variância (ANOVA). $\mathrm{O}$ resultado indicou que não houve diferença significativa entre as faixas etárias para a meta performance-evitação $[F(2,417)=1,289 ; p=0,277]$ e a meta performance-aproximação $[F(2,414)=0,074 ; p=0,929]$. Já para a meta aprender, houve indicação de diferença entre as faixas etárias $[F(2,417)=4,004 ; p=0,019]$. 
Na sequência, recorreu-se ao teste post-hoc de Tukey para identificar quais agrupamentos se diferenciavam na meta aprender, como apontado pela ANOVA. A Tabela 4 apresenta o resultado obtido.

Tabela 4 - Agrupamento por idade e pontuação média na meta aprender

\begin{tabular}{lllc}
\hline Agrupamento por idade & $\mathrm{N}$ & \multicolumn{2}{c}{ Agrupamento por alfa $=0.05$} \\
\hline & & 1 & 2 \\
\hline 25 anos ou mais & 118 & 14.83 & 15.82 \\
Até 19 anos & 133 & & 15.96 \\
De 20 a 24 anos & 169 & & 0.942 \\
$p$ & & 1,000 & \\
\hline
\end{tabular}

Pode-se verificar, na Tabela 4, que o grupo de estudantes da faixa etária com mais de 25 anos se diferenciou dos outros dois grupos etários, porém com uma pontuação média mais baixa. Dessa forma identificou-se que os estudantes mais novos e de idade intermediária diferenciam-se dos mais velhos no fator que avalia a meta aprender.

Quanto à variável área do conhecimento, os estudantes foram divididos em três grupos, a saber, Ciências Exatas, Humanas e Biológicas. As diferenças foram identificadas para os três tipos de metas em função das três áreas do conhecimento. Para isso, realizou-se a análise de variância ANOVA e as diferenças de médias não foram significativas para nenhum dos fatores, isto é, para a performance-evitação $[F(2,417)=0,494 ; p=0,631]$, performance-aproximação $[F(2,414)=2,524$; $p=0,081]$ e aprender $[F(2,417)=0,070 ; p=0,932]$.

\section{Discussão}

O objetivo deste trabalho foi buscar evidências de validade, por meio da análise da estrutura interna dos itens, para uma escala que avalia a motivação para a aprendizagem em universitários. Tendo em vista a busca das propriedades psicométricas da escala EMAPRE-U, foi realizada a análise fatorial para identificar os fatores que compõem a escala e a extração do alfa de Cronbach para avaliar a consistência interna dos fatores e da escala como um todo. Ainda, foram verificadas as diferenças das médias em relação ao sexo ( $t$ de Student), faixa etária e área do conhecimento dos cursos (ANOVA).

A análise fatorial possibilitou o agrupamento dos itens da escala em três fatores, sendo que o Fator 1 corresponde à meta performance-evitação, o Fator 2 à meta performance-aproximação e o Fator 3 à meta aprender, com uma porcentagem de variância total aceitável $(39,41 \%)$. Dos três fatores o que apresentou autovalor mais alto foi o Fator 1, sendo o responsável pela explicação de 19,60\% da variância total.

Quanto ao agrupamento dos itens em torno de cada fator,pode-se notar que os mesmos têm relação com o construto de cada meta. No primeiro fator, meta performance-evitação, agruparam-se dez itens, sendo sete exclusivamente nesse fator e três também com carga no fator 2 . Ao se analisar o conteúdo de cada 
item percebe-se que todos se referem às ações que evitem situações que possam mostrar a incapacidade ou trazer alguma consequência negativa, como por exemplo, "Não participo das aulas para evitar que meus colegas e professores me achem pouco inteligente" e "Não participo dos debates em sala de aula, porque não quero que os colegas riam de mim". Quanto aos três itens que carregaram no Fator 1 e no Fator 2, optou-se por mantê-los no Fator 2, devido ao seu conteúdo ser mais congruente teoricamente com a meta performance-aproximação.

No segundo fator, além dos três itens já mencionados, os outros seis também podem ser interpretados como correspondentes à definição de meta performance-aproximação, cujo foco está no desejo de parecer inteligente e ser melhor do que os outros. Pode-se destacar como exemplos desses itens "É importante para mim, fazer as tarefas melhor que os meus colegas" e "Na minha turma, eu quero me sair melhor que os demais".

Os 12 itens que se agruparam no Fator 3, meta aprender, também estão congruentes com a teoria e se referem ao desejo e interesse em aprender. São exemplos desses itens "Faço minhas tarefas acadêmicas porque estou interessado nelas" e "Uma razão importante pela qual faço as tarefas acadêmicas é porque eu gosto de aprender coisas novas".

Vale mencionar, que o agrupamento dos itens da escala encontrado neste estudo foi similar ao da pesquisa brasileira de Zenorini e Santos (2010a) com alunos do ensino médio, sendo também congruente com os resultados de pesquisas clássicas sobre o tema como as de Elliot e Church (1997) e Elliot e Harackiewicz (1996) e Midgley et al. (1998), que já apontavam para a existência da divisão da meta performance em dois componentes, quais sejam aproximação e evitação. No entanto, houve uma variação na distribuição das cargas fatoriais. No presente estudo, as cargas fatoriais para a meta performance-evitação e meta performan$c e$-aproximação foram maiores, enquanto que para a meta aprender foram menores, quando comparadas ao estudo das referidas autoras.

Quanto à consistência interna, os índices observados para a escala total e para suas subescalas podem ser considerados aceitáveis. A subescala referente à meta performance-evitação foi a que apresentou um índice maior $(0,83)$, enquanto que a meta aprender foi a subescala com o menor índice $(0,72)$. Ao se comparar os índices de consistência interna deste estudo com os encontrados por Zenorini e Santos (2010a), podem-se perceber algumas diferenças. No estudo dessas autoras, o maior coeficiente foi para a meta aprender $(0,80)$, seguida da meta performance-aproximação $(0,76)$ e meta performance-evitação $(0,74)$. Fica a indagação sobre possíveis diferenças entre a precisão interna das subescalas da EMAPRE, a ser explorada em outros estudos que venham a cotejar os escores obtidos com estudantes do ensino médio e com universitários para investigar se essa tendência se mantém.

No que se refere aos índices de correlação entre as subescalas verificou-se que a correlação entre o Fator 1 - meta performance-evitação e o Fator 2 - meta performance-aproximação foi moderada, o que pode ser justificado pelo fato de ambos serem dimensões da meta performance. No entanto, no estudo de Zenorini 
e Santos (2010a) a correlação entre esses dois fatores foi considerada de fraca magnitude $(r=0,14)$. Quanto ao Fator 3 - meta aprender constatou-se que não houve correlação com os demais tipos de metas, visto que os valores foram de baixa magnitude. Esses resultados são congruentes, em parte, com os obtidos na versão da escala para o ensino médio de Zenorini e Santos (2010a), que indicou uma correlação significativa e de forma positiva, com fraca magnitude entre as metas aprender e performance-aproximação $(r=0,13)$. A correlação positiva encontrada entre a meta performance-aproximação e meta performance-evitação também foi coerente com os achados de Elliot e Church (1997), Elliot e Harackiewcz (1996) e de Midgley et al. (1998).

No que se refere à comparação das pontuações médias em relação ao sexo, os resultados apontaram que a média obtida pelos universitários é muito similar tanto na meta performance-evitação quanto na meta aprender. Esses achados diferem dos encontrados em outras pesquisas (BORUCHOVITCH, 2008; GUIMARÃES; BZUNECK; SANCHES, 2002; MIDGLEY; KAPLAN; MIDDLETON, 2001; SOBRAL, 2003; ZENORINI; SANTOS, 2004; ZENORINI; SANTOS; BUENO, 2003; ZENORINI; SANTOS; MONTEIRO, 2011), em que as participantes do sexo feminino mostraram-se mais orientadas à meta aprender e à motivação intrínseca. Já na meta performance-aproximação apareceu uma diferença significativa, sendo que as mulheres obtiveram médias maiores quando comparadas às dos homens. Esse resultado também é divergente de outras pesquisas, como por exemplo, a de Middleton e Midgley (1997), em que os achados apontaram os participantes do sexo masculino com maior tendência para a meta performance-aproximação. Também, com relação a esse aspecto é importante que haja cautela na generalização dos achados, até que outros estudos sejam feitos com a escala em outras amostras brasileiras.

Em se tratando das diferenças nas pontuações médias em relação à faixa etária, o agrupamento por idade mostrou que os estudantes da faixa dos 25 anos ou mais se diferenciam dos de 20 a 24 anos e de até dos de 19 anos, na meta aprender. Como a pontuação do grupo dos estudantes mais velhos mostrou uma média inferior, pode-se supor que os estudantes mais novos e de idade intermediária sejam os que apresentem interesse mais voltado para a aprendizagem, bem como maior persistência diante das dificuldades. Isso pode ser um indicativo de mudança no perfil dos atuais estudantes, pois se pressupõe que na faixa etária de 25 ou mais o estudante já esteja mais "maduro" em relação às suas escolhas e metas de realização, contribuindo dessa forma, para o seu maior envolvimento com as atividades acadêmicas. Vale ressaltar, que esse resultado foi coerente com parte da literatura que aponta um declínio da motivação com o avançar da idade e da escolaridade (LEPPER; CORPUS; YENGAR, 2005; OTIS; GROUZET; PELLETIER, 2005; STIPEK, 1998). Diferentemente dos estudos de Boruchovitch (2008) e Guimarães, Bzuneck e Sanches (2002) que revelaram entre os alunos mais velhos médias mais altas na motivação. É importante salientar mais uma vez a necessidade de novos estudos para conhecer melhor a influência da idade na motivação. 
Outro aspecto explorado foi relativo a possíveis diferenças entre as metas de realização, quando se considerou a variável área de conhecimentos dos cursos. Contudo, não foi encontrado nenhum valor significativo, quando feita essa comparação. Comparações similares não foram encontradas na literatura recuperada sobre o tema.

\section{CONSIDERAÇões FINAIS}

Os resultados encontrados no presente estudo mostraram índices aceitáveis de validade e de precisão para a EMAPRE-U. Quanto às correlações, vale mencionar que o índice moderado encontrado entre a meta performance-evitação e a meta performance-aproximação pode ser um indicativo de que as duas são partes integrantes da meta performance, visto que inclusive três itens apresentaram carga fatorial em ambos os tipos de metas . Enquanto que o fato da meta aprender não se correlacionar com as demais, mostra que essa e as metas performance são aspectos independentes do fenômeno.

Contudo, julga-se necessário destacar algumas limitações encontradas, em especial referente à amostra pesquisada. Embora o número de participantes seja considerado satisfatório para o objetivo pretendido, todos eles são de uma única instituição do ensino superior. Também é interessante comentar sobre a variância explicada do instrumento, que em seu total não chegou a 40\%. Talvez o uso de técnicas estatísticas usadas mais recentemente em pesquisas brasileiras, como o funcionamento diferencial do item (DIF), pudesse auxiliar e explorar outros aspectos da estrutura interna da EMAPRE-U.

Essas considerações não diminuem o potencial de contribuição da investigação aqui apresentada, mas apontam para a necessidade de realização de futuras pesquisas, que permitam não apenas explorar outras evidências de validade. Nesse sentido, vale destacar que estudos de validade de critério, usando outras informações dos alunos, seriam de grande valia para que se pudesse de fato verificar os efeitos das orientações de metas nos alunos em relação ao desempenho acadêmico, por exemplo. Ainda no que se refere a aspectos psicométricos seria relevante explorar a relação dos resultados da EMAPRE-U com outros construtos que têm se mostrado importantes no contexto universitário, tais como a integração e a satisfação acadêmica, entre outros.

Cabe comentar, por fim, que a Teoria de Metas de Realização, que fornece a base teórica para a construção do instrumento aqui descrito, salienta que o ambiente de sala de aula e a atuação do professor são elementos essenciais no desenvolvimento das metas. Sob essa perspectiva, espera-se que os resultados obtidos com o uso da escala possam contribuir para a implementação de práticas educativas que favoreçam a construção do conhecimento, à medida que valoriza o processo de aprendizagem. 


\section{REFERÊNCIAS}

AMES, C. Classrooms: Goals, structures and student motivation. Journal of Educational Psychology, v.84, n.3, p.261-271, 1992.

ANDERMAN, E. M.; MAEHR, M. L. Motivation and schooling in the middle grades. Review of Education Research, v.64, n.2, p.287-309, 1994.

ARCHER, J. Achievement goals as a measure of motivation in university students. Contemporary Educational Psychology, v.19, p.430-446, 1994.

BORUCHOVITCH, E. A motivação para aprender de estudantes em cursos de formação de professores. Educação, v.31, n.1, p.30-38, jan./abr. 2008.

BORUCHOVITCH, E.; BZUNECK, J. A. Motivação para aprender no Brasil: estado da arte e caminhos futuros. In: BORUCHOVITCH, E.; BZUNECK, J. A.; GUIMARÃES, S. É. R. (Org.). Motivação para aprender: aplicações no contexto educativo. Petrópolis: Vozes, 2010. Cap.10, p.231-250.

BUENO, J. M. H. et al. Investigação das propriedades psicométricas de uma escala de metas de realização. Estudos de Psicologia, Campinas, v.24, n.1, p.7987, jan./mar. 2007.

BZUNECK, J. A. Uma abordagem sócio-cognitivista à motivação do aluno: a teoria de metas de realização. Psico-USF, Itatiba, v.4, n.2, p.51-66, 1999.

BZUNECK, J. A. A motivação do aluno orientado à meta aprender. In: BORUCHOVITCH, E.; BZUNECK, J. A. (Org.), A motivação do aluno: contribuições da psicologia contemporânea. 3. ed. Petrópolis: Vozes, 2004. Cap. 3, p.58-77.

CARDOSO, L. R. Uso de estratégias de aprendizagem e suas relações com metas de realização: um estudo no ensino superior. 2002. Dissertação (Mestrado em Educação) - Programa de Pós-Graduação Stricto Sensu em Educação, Universidade Estadual de Londrina, Londrina-PR, 2002.

CLAYTON, K.; BLUMBERG, F.; AULD, D. The relationship between motivation, learning strategies and choice of environment whether traditional or including an online component. British Journal of Educational Technology, v.41, n.3, p.349-364, 2010.

ELLIOT, A. J.; CHURCH, M. A. A hierarchical model of approach and avoidance achievement motivation. Journal of Personality and Social Psychology, v.72, p.218 232, 1997.

ELLIOT, A. J.; HARACKIEWICZ, J. M. Approach and avoidance achievement goals and intrinsic motivation: a mediational analysis. Journal of Personality and Social Psychology, v.70, n.3, p.461-75, 1996. 
ELLIOT, A. J.; McGREGOR, H. A.; GABLE, S. Achievement goals, study strategies, and exam performance: A meditational analysis. Journal of Educational Psychology, v.91, p.549-563, 1999.

GUIMARAES, S. É. R.; BZUNECK, J. A.; SANCHES, S. F. Psicologia educacional nos cursos de licenciatura: a motivação dos estudantes. Psicologia Escolar e Educacional, v.6, n.1, p.11-19, 2002.

LEPPER, M. R.; CORPUS, J. H.; IYENGAR, S. Intrinsic and extrinsic motivation in the classroom: age differences and academic correlates. Journal of Educational Psychology, v.97, n.2, p.184-196, 2005.

MAEHR, M. L.; MIDGLEY, C. Enhancing student motivation: a school wide approach. Educational Psychologist, v.26, n.3/4, p.399-427, 1991.

MIDDLETON, M. J.; MIDGLEY, C. Avoiding the demonstration of lack of ability: An underexplored aspect of goal theory. Journal of Education Psychology, v.89, n.4, p.710-728, 1997.

MIDGLEY, C.; KAPLAN, A.; MIDDLETON, M. Good for what, for whom, under what circumstances, and at what cost? Journal of Educational Psychology, v.93, n.1, p.77-86, 2001.

MIDGLEY, C. et al. The development and validation of scales assessing student's achievement goals orientations. Contemporary Educational Psychology, n.23, p.113 131, 1998.

OTIS, N.; GROUZET, M. E. F.; PELLETIER, L. G. Latent motivational change in an academic setting: a 3-year longitudinal study. Journal of Educational Psychology, v.97, n.2, p.170- 183, 2005.

PINTRICH, P. R.; SCHUNK, D. H.. Motivation in education: theory, research, and applications. Englewood Cliffs, N. J.: Prentice Hall, 1996.

RYAN, R. M.; DECI, E. L. Self-determination theory and the facilitation of intrinsic motivation, social development, and well-being. American Psychologist, v. 55, p. $68-78,2000$.

SOBRAL, D. T. Motivação do aprendiz de medicina: uso da Escala de Motivação Acadêmica. Psicologia: Teoria e Pesquisa, v.19, n.1, p.25-31, jan./abr. 2003.

STEFANO, S. R. As orientações motivacionais em cursos de Administração: um estudo comparativo entre alunos de instituição pública e privada. 2002. Dissertação (Mestrado em Administração) - Programa de Pós-Graduação Stricto Sensu em Administração, Universidade Estadual de Londrina, Londrina-PR, 2002.

STEINMAYR, R.; SPINATH, B. The importance of motivation as a predictor of school achievement. Learning and Individual Differences, n.19, p.80-90, 2009. 
STIPEK, D. J. Motivation to learn: from theory to practice. Englewood Cliffs, NJ: Preintice Hall, 1998.

VALLE, A. et al. Metas acadêmicas, estrategias cognitivas y estrategias de autorregulación del estúdio. Psicothema, v.18, n.2, p.165-170, 2006.

VALLE, A. et al. Academic goals and learning quality in higher education students. The Spanish Journal of Psychology, v.12, n.1, p.96-105, 2009.

VROOM, V.H. Work and Motivation. New York, NY: John Wiley and Sons, 1964.

WEINER, B. An attributional theory of achievement motivation and emotion. Psychological Review, v. 92, n.4, p. 548-573, 1985.

WEINER, B. History of motivational research in education. Journal of Educational Psychology, v.82, n.4, p.616-622, 1990.

WOLTERS, C. A.; YU, S. L.; PINTRICH, P. R. The relation between goal orientation and student's motivational belefies and self-regulated learning. Learning and individual Differences, n.8, p.211-238, 1996.

ZENORINI, R. P. C.; SANTOS, A. A. A. A motivação e a utilização de estratégias de aprendizagem em universitários. In: MERCURI, E.; POLYDORO, S. A. J. Estudante universitário: característica e experiências de formação. Taubaté: Cabral, 2004. p. 67-86.

ZENORINI, R. P. C.; SANTOS, A. A. A. Escala de metas de realização-versão para universitários. Relatório técnico. Programa de Pós-graduação stricto-sensu da Universidade São Francisco, 2008.

ZENORINI, R. P. C.; SANTOS, A. A. A. Escala de metas de realização como medida da motivação para aprendizagem. Interamerican Journal of Psychology, v.44, n.2, p.291-298, 2010a.

ZENORINI, R. P. C.; SANTOS, A. A. A. Teoria de metas de realização: fundamentos e avaliação. In: BORUCHOVITCH, E.; BZUNECK, J. A.; GUIMARÃES, S. E. R. (org.). Motivação para aprender: aplicações no contexto educativo Petrópolis: Vozes, 2010b. Cap. 4, p.99-125.

ZENORINI, R. P. C.; SANTOS, A. A. A.; BUENO, J. M. H. Escala de avaliação das metas de realização: estudo preliminar de validação. Avaliação Psicológica, v.2, n.2, p.165-173, 2003.

ZENORINI, R. P. C.; SANTOS, A. A. A.; MONTEIRO, R. M. (2011). Motivação para aprender: relação com o desempenho de estudantes. Paidéia, v. 21, n. 49, p. 157-164, 2011.

Recebido em: 21 de janeiro de 2011

Aceito em:13 de março de 2013 